\title{
Evidence for Task-Dependent Memory Dysfunction in the Aged Monkey
}

\author{
Peter R. Rapp and David G. Amaral \\ The Salk Institute, La Jolla, California 92037
}

\begin{abstract}
Experimentally naive adult (9-11 years old) and aged (approximately 22-26 years old) female rhesus monkeys were evaluated on 3 neuropsychological tests of memory function. Aged monkeys were impaired in a delayed response test of visuospatial memory when the retention interval of the task was increased from 0 to 10 sec. These animals performed as well as younger subjects, however, at very short delays $(0$ and $1 \mathrm{sec}$ ), when the memory demands of the task were minimal. The same subjects were then trained in a delayed nonmatching to sample (DNMS) test of visual object recognition memory. Although they required significantly more training than the younger subjects to learn the nonmatching principle of the task, aged animals were only minimally impaired when recognition memory was tested at retention intervals ranging from $10 \mathrm{sec}$ to $22 \mathrm{hr}$. In contrast to their relatively intact performance on the object recognition task, aged monkeys were dramatically impaired in a second version of DNMS that required subjects to remember the temporal order in which objects were presented. These findings support the view that certain memory functions are differentially susceptible to age-dependent deterioration. Since neuropsychological studies in young subjects demonstrate that different brain regions make relatively specific contributions to learning and memory, the task-dependent deficits observed in the aged monkey are important for determining which neural structures mediate age-dependent cognitive dysfunction. According to this perspective, aged monkeys were impaired on tasks known to be sensitive to prefrontal cortical damage, but the same animals performed well on a DNMS procedure that subjects with medial temporal lobe damage fail. These results suggest that prefrontal cortical dysfunction may mediate prominent aspects of age-dependent cognitive impairment in the monkey.
\end{abstract}

There is a growing consensus in modern neuropsychology that memory is a multicomponent process rather than a unitary function. An emerging parallel view in research on age-related memory dysfunction is that mnemonic processes are differentially susceptible to age-dependent deterioration. Some behavioral tests that clearly require memory are performed normally by aged subjects and the presence or absence of age-related deficits appears to depend on the specific information-process-

\footnotetext{
Received Feb. 7, 1989; revised Apr. 3, 1989; accepted Apr. 3, 1989.

This work was supported by NIH grants NS 16980 and MH 41479. We thank Ms. Karen Suter for her help in behavioral testing and Dr. Stuart Zola-Morgan for his advice during this project.

Correspondence should be addressed to Dr. Peter R. Rapp, The Salk Institute P.O. Box 85800, San Diego, CA 92138.

Copyright $(\odot 1989$ Society for Neuroscience $0270-6474 / 89 / 103568-09 \$ 02.00 / 0$
}

ing demands of the tasks used. Studies on normal aging in humans, for example, indicate that immediate memory as assessed by digit span remains intact (Ferris et al., 1980). Age-dependent deficits often are observed, however, when memory for recent events is tested over longer retention intervals (Adamowicz, 1976; Flicker et al., 1984; Rabinowitz, 1984). Moreover, memory for specific classes of information (e.g., spatial, temporal, verbal) may be differentially compromised during human aging (Perlmutter et al., 1981; Park et al., 1983). Another type of selective deficit in aging was demonstrated by Light et al. (1986), who found that aged subjects exhibit intact priming for recently presented words even though recognition memory for this same material is impaired. Even in patients exhibiting the dramatic cognitive deterioration that accompanies Alzheimer's disease, normal motor learning (Eslinger and Damasio, 1986; Heindel et al., 1989) and a relative preservation of very remote memories (Beatty et al., 1988; Sagar et al., 1988) have been observed.

Task-dependent behavioral impairments also appear to be a prominent feature of aging in nonhuman species. Aged rats, for example, are dramatically impaired across testing procedures that require the acquisition and utilization of spatial information (Barnes, 1979; Barnes et al., 1980; Gage et al., 1984; Rapp et al., 1987; Pelleymounter et al., 1987). Performance on nonspatial versions of some of these same tasks, however, remains relatively intact (Barnes et al., 1980; Rapp et al., 1987). Although the effects of aging on cognitive function in the monkey have not been studied extensively, the available evidence suggests that aged monkeys are impaired on only a subset of behavioral testing procedures that require memory. Aged monkeys exhibit robust impairments on tasks that are dependent on recently presented visuospatial information (Medin, 1969; Bartus et al., 1978; Arnsten and Goldman-Rakic, 1985a; Walker et al., 1988), but they perform normally in simple 2-choice discrimination procedures that require the acquisition and retention of color, object or pattern information (Bartus et al., 1979; Arnsten and Goldman-Rakic, 1985a; Moss et al., 1988; Walker et al., 1988).

One important outcome of characterizing impaired versus preserved function in aging is the insight this information can provide concerning the neural systems that mediate age-dependent cognitive dysfunction. Neuropsychological findings emphasize that different regions of the brain make relatively specific contributions to memory. Studies of amnesic patients and monkeys with experimental brain damage demonstrate that the hippocampal formation (Scoville and Milner, 1957; Mishkin, 1978; Zola-Morgan and Squire, 1985a; Zola-Morgan et al., 1989) and certain diencephalic structures (Victor et al., 1971; Squire and Moore, 1979; Aggleton and Mishkin, 1983a, b; Zola-Morgan and Squire, 1985b) play a critical role in the formation of factbased, context-dependent memories. The amygdala, in contrast, 
seems to be particularly important for memory that requires the association of information from different sensory modalities (Murray and Mishkin, 1985; Gaffan and Harrison, 1987). Other cognitive processes, including the organization of information in both spatial and temporal contexts, appear to be particularly dependent on prefrontal cortical function (Milner and Petrides, 1984; Goldman-Rakic, 1987; Schacter, 1987). Since different brain regions appear to make rather spccialized contributions to learning and memory in young subjects, specific types of cognitive impairment in aged subjects may reflect the functional status of those brain regions that mediate the component processes of memory. Based on this neuropsychological perspective, the present experiments evaluated the performance of young and aged monkeys on 3 behavioral tasks that are differentially dependent on the specific contributions of different brain regions to learning and memory.

\section{Materials and Methods}

Subjects. Nine experimentally naive female rhesus monkeys (Macaca mulatta) served as subjects in these experiments. Five aged subjects were obtained as retired breeders from the New Iberia Research Center in Louisiana and were estimated to be between 22 and 26 years old at the time they were behaviorally tested (6/1987-1/1989). These animals were feral born and entered the Louisiana facility during the period 1967-1970. At this time, all animals were of sufficient maturity to breed successfully. Since reliable breeding is not typical of female rhesus monkeys younger than 4 years of age (Goo, 1986), the estimates given above represent a minimum estimated age range for the older subjects.

Four younger monkeys were obtained from a breeding facility at the University of Montana. These animals were born in captivity and were documented to be 9-11 years old at the time they were behaviorally tested (1/1988-1/1989). The 2 groups described above served as the aged ( $n=5$, mean weight $8.4 \mathrm{~kg}$, range $6.4-9.5 \mathrm{~kg}$ ) and young adult $(n$ $=4$, mean weight $6.0 \mathrm{~kg}$, range 4.3-7.9 $\mathrm{kg}$ ) samples for these studies. Although longevity figures have not been fully established, estimates suggest that the average life span of the rhesus monkey is probably less than 30 years (Davis, 1985; Tigges et al., 1988). The two groups chosen for these studies therefore represent young, fully mature subjects and animals approaching the end of their expected life span

It is important to note that the groups used here were well matched in terms of behavioral history and conditions of housing prior to their arrival at the Salk Institute. All subjects were maintained as part of long-term stable breeding programs and were not used in any research prior to the present experiments. Before their arrival at the Salk Institute, all subjects were group housed in well-socialized harems with access to both indoor and outdoor runs.

On arrival at the Salk Institute, subjects were singly housed and maintained on Wayne Monkey Diet supplemented regularly with fresh fruit. Feeding took place once a day following behavioral testing. Subjects were not specifically food deprived, but rations were adjusted to maintain stable levels of performance. Water was available ad libitum in the home cage throughout all experiments. The vivarium was maintained on a 12-hr light-dark cycle (lights on at $0600 \mathrm{hr}$ ) at an average ambient temperature of $23^{\circ} \mathrm{C}$. Behavioral testing was conducted between 0800 and $1700 \mathrm{hr}$ during the light phase of the cycle. All animals received periodic comprehensive physical examinations, and subjects from both groups remained in good health throughout these experiments.

Apparatus. All behavioral testing was conducted in a modified Wisconsin General Test Apparatus (WGTA) (Harlow and Bromer, 1938). During testing, the subject was transferred from the home cage to a sound-attenuating chamber $(71.1 \mathrm{~cm}$ high $\times 58.4 \mathrm{~cm}$ wide $\times 81.3 \mathrm{~cm}$ deep). This chamber contained an opening $(24.1 \mathrm{~cm}$ high $\times 39.5 \mathrm{~cm}$ wide) with vertical bars separated by $5.0 \mathrm{~cm}$ that faced the stimulus tray of the WGTA. The stimulus tray contained 3 food reward wells ( $2.5 \mathrm{~cm}$ in diameter and $0.6 \mathrm{~cm}$ deep), separated by $12 \mathrm{~cm}$ from center to center and fixed $12.7 \mathrm{~cm}$ in front of the subject chamber. A superstructure supporting 3 experimenter-controlled screens was used to isolate the stimulus tray and experimenter from the subject as needed. A clear Plexiglas screen could be lowered between the caged monkey and the food wells of the stimulus tray to physically but not visually isolate the subject during testing. A second, opaque screen could be lowered to both physically and visually isolate the monkey from the stimulus tray. The third screen was situated between the stimulus tray and the experimenter and contained a one-way mirror so that the tester could observe the animal's performance undetected. A white-noise generator was used to mask extraneous sound during all phases of testing.

\section{Behavioral testing procedures}

Pretraining. Pretraining was initiated no sooner than 10 weeks after the animals' arrival at the Salk Institutc. During this acclimation period, food and water intake rapidly stabilized. All subjects quickly learned to accept fruit from the hand of the experimenter, and no subjects maintained overt signs of stress or deteriorating health.

Following this acclimation period and before any formal testing, subjects were shaped to displace an object and retrieve food rewards from the wells of the WGTA. Food preferences were noted on the basis of which rewards were chosen first or most often, and an individual animal's preferred reward was used in later phases of testing. Initially, small piles of peanuts and raisins were presented over the 3 wells of the stimulus tray, and the subject was given the opportunity to eat. When animals came to respond quickly in this condition, reinforcement was gradually reduced until subjects had to displace an object covering one of the food wells in order to retrieve a single reward. When stable levels of performance were achieved in this final phase of prctraining, formal training was initiated.

Delayed response. Subjects were first trained in a delayed response task (DR) using the method of direct baiting. A schematic representation of this procedure is illustrated in Figure 1. Training was conducted in 2 phases: basic training and testing with delays. A trial was initiated when the opaque screen separating the subject from the stimulus tray was raised. With the transparent Plexiglas screen in place between the subject and the stimulus tray, one of the lateral food wells was baited while the monkey watched. The use of a transparent screen in this task allowed the stimulus tray to be baited directly in front of the caged monkey. This procedure was intended to minimize the influence of potential age-dependent differences in visual acuity on DR performance. The lateral wells were then covered with identical gray plastic plaques ( $7.6 \mathrm{~cm}$ square $\times 0.9 \mathrm{~cm}$ high). During basic training, the clear screen was raised immediately after the food wells were covered, and the monkey was allowed to respond by displacing one of the plaques. When the subject chose the correct well, it was permitted to retrieve and eat the reward. Monkeys were provided with 20 trials/d, using a 20 -sec intertrial interval. Across trials, the left and right wells of the stimulus tray were baited equally often according to a pseudorandom sequence. Basic training was continued until subjects met a $90 \%$ learning criterion by committing no more than 10 errors in 100 consecutive trials.

After reaching criterion levels of performance on the basic version of the task, testing was continued in a similar fashion except that a variable retention delay was imposed between the baiting and response phase of each trial. On these trials, the subject watched through the clear screen as one of the lateral food wells was baited. Both lateral wells were then covered with gray plaques as in the basic version of the task. The opaquc screen was then lowered for a period of $1,5,10,15$, or $30 \mathrm{sec}$, after which both the opaque and clear screens were raised to allow the subject to respond. Subjects were tested for 20 trials/d, with each delay interval occurring 4 times/d according to a pseudorandom sequence. DR testing with delays continued for 13 sessions for a total of 52 trials at each of the 5 delay intervals. The left and right wells of the stimulus tray were baited equally often in each session, and a $20 \mathrm{sec}$ intertrial interval was used for all test sessions.

Delayed nonmatching to sample with trial unique objects. Following DR training, visual recognition memory was tested using the delayed nonmatching to sample with trial unique objects (DNMS-TU) procedure illustrated in Figure 3. Each trial in the DNMS-TU task consisted of a sample and a recognition phase. A trial was initiated when the opaque screen of the WGTA was raised to reveal a single object covering the baited central well of the stimulus tray. The monkey was then permitted to displace the object and retrieve the reward. Following this sample presentation, the opaque screen was lowered, and a novel object was used to conceal a reward in one of the lateral food wells. At the same time, the sample object was placed over the remaining empty lateral well. The recognition phase of each trial was initiated when the opaque screen was raised to reveal both the novel object and the sample object. The subject was then permitted to displace one of the objects and, if the novel object was chosen, retrieve and eat the reward. A new pair of objects that the subject had not seen previously was used on 
every trial. Reward was therefore contingent on the monkey's recognizing and avoiding the sample object in the recognition phase of each trial.

The objects used in this task were chosen from a collection of over 520 items that differed widely in shape, color, and texture. Although subjects saw individual objects a number of times over the course of DNMS-TU testing, 2 objects were never presented as a pair more than one time for each subject. The number of objects used in this procedure allowed individual items to be repeated no more often than approximately once every 2.5 weeks of testing.

Subjects were initially trained in the DNMS-TU procedure with a $10-\sec$ delay between the sample and recognition phase of each trial. Each daily test session consisted of 20 trials with a $30-\mathrm{sec}$ intertrial interval. The correct (i.e., novel) object in the recognition phase of each trial appeared over the left or right food well equally often, and the object in each pair that was used as the sample was balanced across animals within a group. Subjects were tested in this fashion using a 10sec delay until reaching a $90 \%$ learning criterion by committing no more than 10 errors in 100 consecutive trials. When criterion performance was achieved with a 10 -sec delay, the memory demands of the task were successively increased by lengthening the delay between the sample and recognition phase of each trial from $15 \mathrm{sec}$ to $22 \mathrm{hr}$. A total of 100 trials ( 20 trials/d) was provided at each of the following delays: $15 \mathrm{sec}$, $30 \mathrm{sec}, 1 \mathrm{~min}$, and $2 \mathrm{~min}$. A total of 50 trials $(5 \mathrm{trials} / \mathrm{d})$ was provided at a 10 -min delay. Finally, a total of 30 trials each $(1 \mathrm{trial} / \mathrm{d})$ was conducted at delays of $2 \mathrm{hr}$ and $22 \mathrm{hr}$.

Delayed nonmatching to sample with repeated objects. Following DNMS-TU testing, subjects were trained on a delayed nonmatching to sample procedure with repeated objects (DNMS-RO). Testing was conducted in a similar fashion in both DNMS-TU and DNMS-RO except that in DNMS-RO, the same pair of objects was used on every trial throughout training, and 1 of these 2 objects appeared as the sample on each trial according to a pseudorandom sequence (see Fig. 5). Many of the task demands were common to both versions of DNMS but when the same pair of objects is presented on every trial (i.e., DNMS-RO), animals are required to make a temporal discrimination in order to perform accurately. That is, since the same 2 objects are presented on every trial, correct performance on the recognition phase of DNMS-RO depends on the subject remembering which of the 2 objects was presented most recently as the sample.

Subjects were initially trained in the DNMS-RO procedure with a 10 -sec delay between the sample and recognition phase of each trial. Daily test sessions consisted of 20 trials with a 30 -sec intertrial interval. The correct object in the recognition phase of each trial appeared over the left or right food well equally often, and the object used as the sample on each trial was balanced across animals within a group. The same pair of objects was used for all animals throughout training. Subjects were tested using a 10 -sec delay until reaching a $90 \%$ learning criterion by committing no more than 10 errors in 100 consecutive trials. Subjects that failed to reach criterion within 1500 trials were trained in a remedial procedure in which the sample object was presented twice in succession before the recognition phase of each trial. The sample object was baited only 1 of the 2 times it was presented according to a pseudorandom sequence. Subjects were tested in this manner until they reached the criterion level of performance or for a total of 300 trials. In either case, subjects were subsequently tested for 100 trials using a single sample presentation and a 10-sec delay interval (i.e., the basic DNMS-RO training procedure used for all animals). Following training with a 10sec delay, the memory demands of the task were successively increased by lengthening the delay between the sample and recognition phase of each trial from 15 to 30 to $60 \mathrm{sec}$. A total of 100 trials ( 20 trials/d) was provided at each delay.

\section{Results}

Prior to formal testing, the general behavior of young and aged subjects was observed on a daily basis. All monkeys readily acclimated to single housing conditions without overt signs of stress, and food and water intake stabilized to normal levels within 2 weeks of the animal's arrival. In their home cages, aged subjects tended to exhibit somewhat less spontaneous activity, but no gross age-related differences were noted in general reactivity or responsiveness to environmental stimuli. Pretraining also failed to reveal gross behavioral differences between young
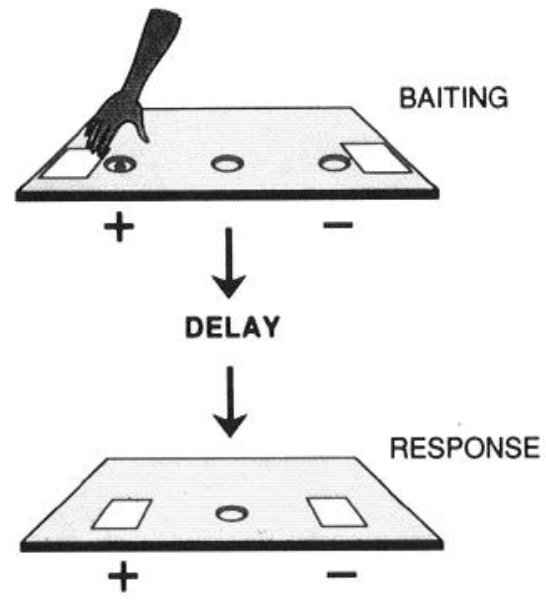

Figure 1. Schematic representation of the delayed response task (DR). Subjects watched while the experimenter baited one of the food wells of the WGTA ( + , baited; - , unbaited). Both wells were immediately covered with identical plaques. Subjects were initially trained to a learning criterion with no delay. Memory for the reward location was subsequently tested with a variable retention interval of 1-30 sec. The intertrial interval was $20 \mathrm{sec}$ throughout training.

and aged monkeys. Although some of the aged animals appeared less dexterous than younger subjects in retrieving rewards from the food wells of the WGTA, all subjects achieved equivalent stable levels of performance during pretraining.

\section{Delayed response}

The number of trials subjects required to achieve a $90 \%$ correct learning criterion in the DR task with a 0 -sec delay is shown in the left panel of Figure 2. One subject in the young group would not reliably observe the food well being baited at the beginning of each trial, and data from this animal were therefore excluded from analysis. All of the remaining subjects met the $90 \%$ correct learning criterion, and the difference in the median performance of young and aged groups during this phase of DR testing was not statistically reliable. Although the average performance of the 2 groups did not differ during basic DR training, withingroup variability in performance was clearly greater among the aged animals. Two aged animals outperformed all of the young subjects, making fewer than 10 errors within the first 100 trials of testing, and 2 aged monkeys took longer to reach criterion than any of the young subjects. Only 1 aged monkey achieved the learning criterion within the range of scores for the young group.

Although young and aged monkeys achieved equivalent levels of response accuracy with a 0 -sec delay in the DR task, the performance of the aged subjects was dramatically and disproportionately affected during DR testing with longer delays. These data are shown in the right panel of Figure 2 which represents the average percent correct achieved by the young and aged groups across trials at delays of $0,1,5,10,15$, and $30 \mathrm{sec}$. Scores for the 0 -sec delay condition are the percent correct achieved during the criterion run of 100 trials on the basic version of the DR task. Statistical evaluation of these results using a two-way ANOVA with performance across delays as a within-subject variable revealed significant main effects of age $[F(1,6)=7.6$, $p=0.03]$, delay $[F(5,30)=13.2, p<0.001]$, and a significant age $\times$ delay interaction $[\mathrm{F}(5,30)=2.4, p=0.05]$. The significant main effects of age and delay indicate that the performance of 

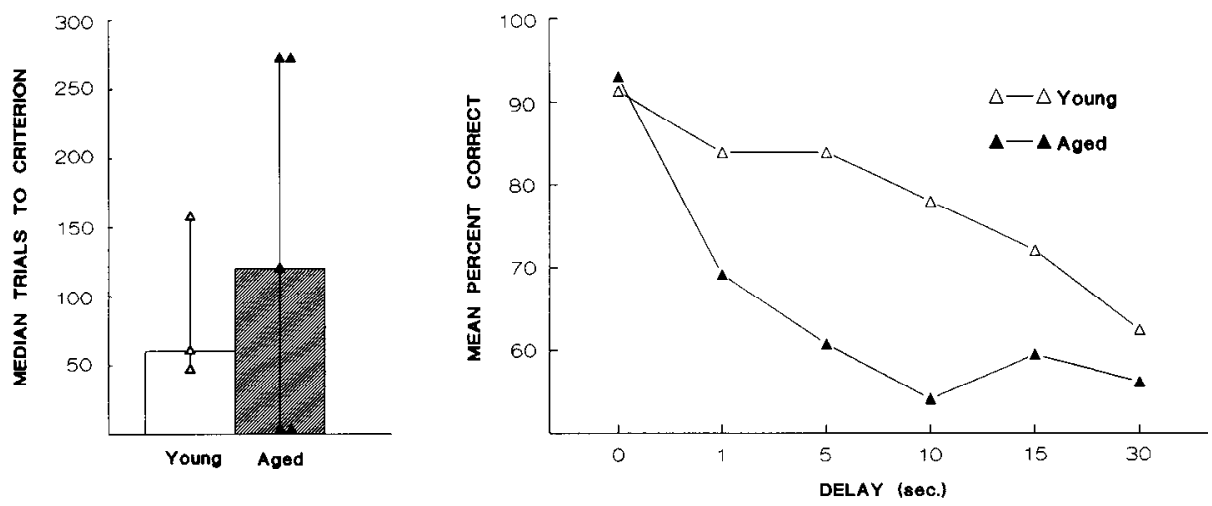

Figure 2. Performance of young ( $n-$ $3)$ and aged $(n=5)$ monkeys on the delayed response task (DR). Left. Median number of trials subjects required to achicve a $90 \%$ correct learning criterion with no delay. Symbols represent the learning scores for individual animals. Right, Average percent correct of the young and aged groups during DR testing with delays. the aged group was impaired relative to the younger group and that the performance of both groups deteriorated at longer delays. Importantly, the age $\times$ delay interaction indicates that the aged subjects were relatively more affected by increasing delays than the younger animals. Subsequent comparisons revealed that the mean performance of the 2 groups was equivalent at 0 - and 1-sec delays but that the aged monkeys were significantly impaired at $5-\sec [t(6)=5.2, p=0.002]$ and $10-\sec [t(6)=3.6$, $p=0.01]$ delay intervals. In fact, no individual aged subject performed within the range of scores obtained by the young animals at these latter delays, and, as a group, aged monkeys did not perform significantly above chance at any delay longer than $5 \mathrm{sec}$. DR performance also began to deteriorate somewhat at 15- and 30-sec delays in the young group, as indicated by the lack of significant group differences in performance at these 2 longest delays.

\section{Delayed nonmatching to sample with trial unique objects}

Aged subjects required significantly more trials than young animals to reach a $90 \%$ correct response criterion in the DNMSTU task with a 10-sec delay (Mann-Whitney $U, p=0.02$ ). These results are illustrated in the left panel of Figure 4. As during initial acquisition of the DR task, within-group variability in performance during basic training in the DNMS-TU task was clearly greater among the aged subjects. One aged subject did not reach the learning criterion within 1330 trials. This monkey was trained for 100 additional trials in a remedial version of the DNMS-TU task in which the sample object was presented twice in succession before the recognition phase of each trial. The sample object was baited only 1 of the 2 times it was presented during this procedure. In all other respects, training was conducted as described for the rest of the animals. During this double sample presentation procedure, performance improved to criterion levels (i.e., $93 \%$ correct in the 100 trials of remedial training). Moreover, when this subject was subsequently tested for 100 trials with a single presentation of the sample object (i.e., the basic version of the task used for all subjects), performance was maintained at $93 \%$ correct. In contrast to this animal's protracted rate of acquisition, 1 aged monkey reached the criterion level of performance within the range of scores exhibited by younger subjects. In summary, although aged monkeys as a group required significantly more trials to learn the DNMS-TU task with a 10-sec delay, subjects in both age groups eventually achieved equivalent levels of performance in this version of the task.

After reaching criterion levels of performance on the basic version of DNMS-TU, recognition memory was tested across delays of $15 \mathrm{sec}$ to $22 \mathrm{hr}$. These data are shown in the right panel of Figure 4. Scores for the 10 -sec delay condition represent the average percent correct attained by each group during the criterion run of 100 trials in the basic version of the task. Although aged subjects were significantly impaired in learning the DNMS-TU task initially, after mastering the nonmatching principle, the older monkeys performed only slightly worse than younger animals across memory delays as long as $22 \mathrm{hr}$. The average performance of the young and aged groups differed by less than 10 percentage points during all phases of testing with delays. The effect of increasing memory demands was evident in both groups as a decrease in recognition accuracy with increasing delay length. This pattern of results was reflected in the statistical analysis which revealed only a significant main effect of delay $[F(7,49)=45.8, p<0.001]$. The main effect of age approached but did not reach significance $[F(1,7)=4.7, p=$ $0.06]$, and there was no indication of a delay $\times$ age interaction. The lack of a significant interaction in these results is particularly important and indicates that, in contrast to performance on the DR task, age-dependent DNMS-TU impairments did not emerge when the memory demands of the task were increased. The borderline significant main effect of age reflects the modest difference in the performance of young and aged subjects across delays. For example, the largest magnitude impairment seen in any aged subject relative to the average performance of the young group was an $18 \%$ deficit at a 2 -hr delay. In contrast, at least 2 aged subjects performed within the range of scores achieved by the young monkeys at each of the delays tested.

\section{Delayed nonmatching to sample with repeated objects}

Only 3 monkeys in each age group learned to perform accurately within 1900 trials of DNMS-RO training using a 10-sec retention interval, and data for the remaining 3 subjects ( 2 aged and 1 young monkey) were, therefore, excluded from analysis. One young monkey performed at only $83 \%$ correct (i.e., $7 \%$ below criterion) even after extensive remedial training using the double sample presentation procedure. This animal consistently performed well above chance, however, even when the memory demands of the task were substantially increased. Since initial learning was somewhat inferior in this subject, including this animal's data in the following analysis provides a conservative test of whether DNMS-RO performance is sensitive to agedependent deterioration. Moreover, all effects that are identified as statistically reliable remain so when this subject's performance is excluded from analysis.

Acquisition data for the animals who learned the DNMS-RO task are presented in the left panel of Figure 6. Although DNMS- 


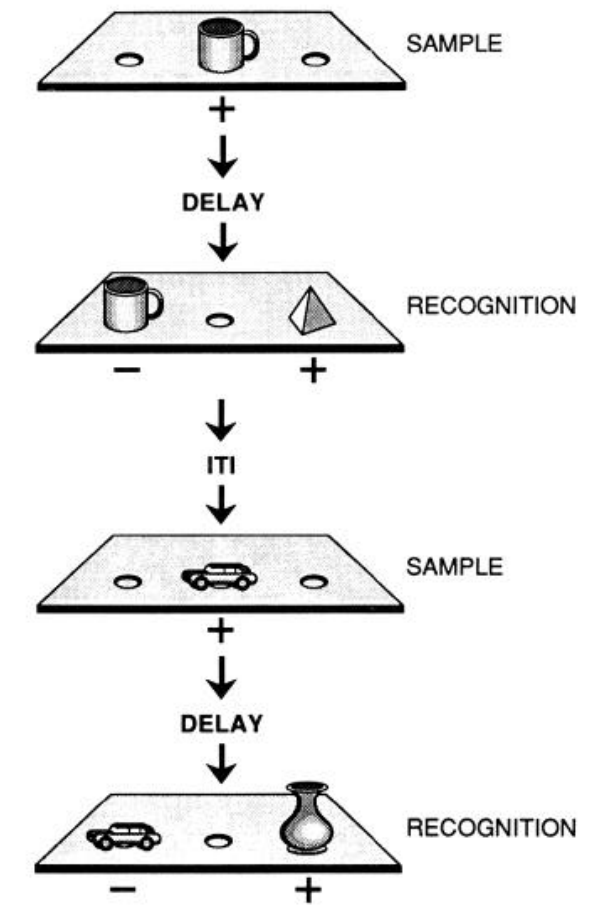

Figure 3. Schematic representation of the delayed nonmatching to sample task with trial unique objects (DNMS-TU). Correct performance required subjects to recognize and avoid a sample object after some delay interval ( + , baited; - , unbaited). A pair of objects that subjects had never seen were used on every trial. Monkeys initially were trained to a learning criterion with a 10 -sec delay. Memory for the sample object was subsequently tested across delays up to $22 \mathrm{hr}$. The intertrial interval $(I T I)$ was 30 sec throughout DNMS-TU training.

RO was clearly the more difficult of the 2 DNMS procedures used here (i.e., compare acquisition data in Figs. 4 and 6), agedependent differences were not observed in the number of trials subjects required to achieve criterion levels of performance during DNMS-RO training.

Following training to criterion on DNMS-RO with a 10 -sec delay, the memory demands of the task were manipulated by increasing the retention interval between the sample and recognition phase of each trial from 15 to $60 \mathrm{sec}$ (Fig. 6, right). Data presented for the 10-sec delay interval represent the average performance of groups during the final 100 training trials in the basic version of the task. Although the performance of the groups was well matched at this shortest delay, recognition accuracy in aged subjects dropped dramatically and disproportionately as the retention interval was increased. This pattern of results was reflected in the statistical evaluation, which revealed significant main effects of age $[F(1,4)=225.3, p<0.001]$ and delay $[F(3,12)=39.3, p<0.001]$, and a significant age $\times$ delay interaction $[F(3,12)=12.2, p<0.001]$. These findings indicate that although the performance of the aged group was impaired relative to younger subjects, the degree of this deficit was exacerbated as a function of the increasing memory demands of the task. Subsequent comparisons confirmed that although recognition accuracy was equivalent at the 10- and 15sec delays, the aged group was significantly impaired during testing at the 2 longer retention intervals [30-sec delay $t(4)=$ $4.3, p=0.01 ; 60$-sec delay $t(4)=13.3, p<0.001]$. In fact, aged subjects as a group performed near chance levels of accuracy at the 60 -sec retention interval. These effects appear to be highly reliable, since no aged subject performed within the range of scores achieved by younger animals at the 2 longer delay intervals. In addition, within-group variability was quite low, with all aged subjects performing within 4.0 percentage points of their group mean at the 30 - and 60 -sec delay intervals.

\section{Discussion}

The major finding of the present study is that memory impairment in the aged monkey is clearly task-dependent. Aged monkeys that exhibit robust deficits in a delayed response task and a delayed nonmatching to sample task with repeated objects are, at most, only mildly impaired in a test of object recognition memory (i.e., DNMS-TU). This task-dependence suggests that specific cognitive processes may be differentially susceptible to age-dependent deterioration. Since neuropsychological studies in young subjects demonstrate that different brain regions make relatively specific contributions to learning and memory, the findings reported here provide a basis for predicting which brain regions are likely to mediate cognitive decline in aging. The value of this approach is that postmortem analyses of the brains from behaviorally characterized subjects will permit a direct evaluation of the relationship between the severity of regional neural alterations and cognitive impairment in aging.

Results from the DR task are consistent with a number of earlier reports demonstrating that aged monkeys are impaired in this testing procedure (Medin, 1969; Bartus et al., 1978; Walker et al., 1988). In a series of studies, Bartus et al. found that rhesus monkeys as young as 18-22 years old show marked delaydependent deficits in an automated, subject-paced DR task (reviewed in Dean and Bartus, 1988). The finding that aged mon-
Figure 4. Performance of young $(n=$ 4) and aged $(n=5)$ monkeys on the delayed nonmatching to sample task with trial unique objects (DNMS-TU). Lefi, Median number of trials subjects required to achieve a $90 \%$ correct learning criterion with a 10-sec delay. Symbols represent the learning scores for individual animals. Right, Average percent correct of the young and aged groups during DNMS-TU testing with delays of $10 \mathrm{sec}$ to $22 \mathrm{hr}$.
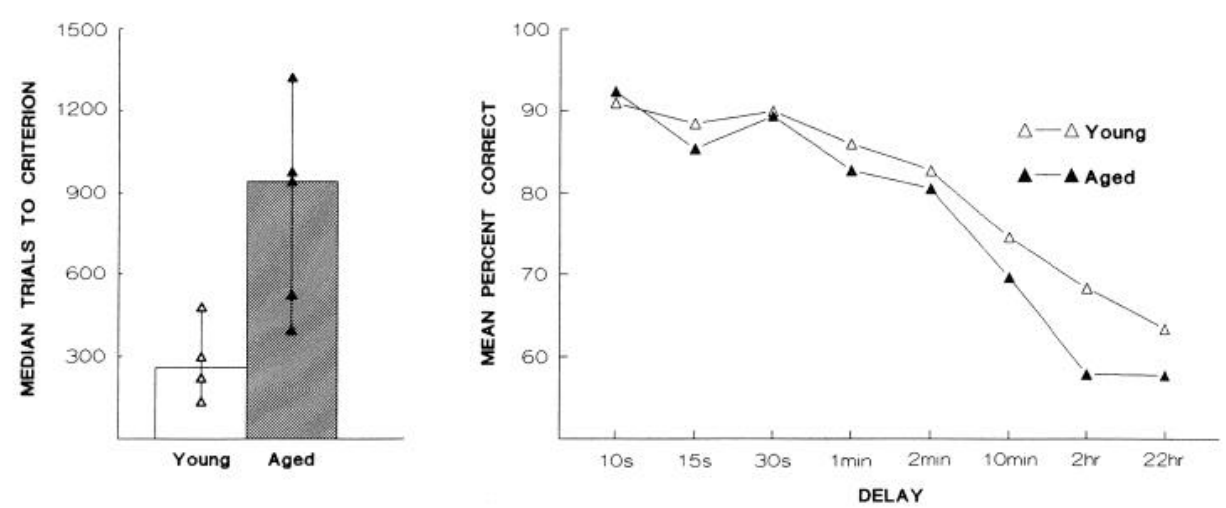


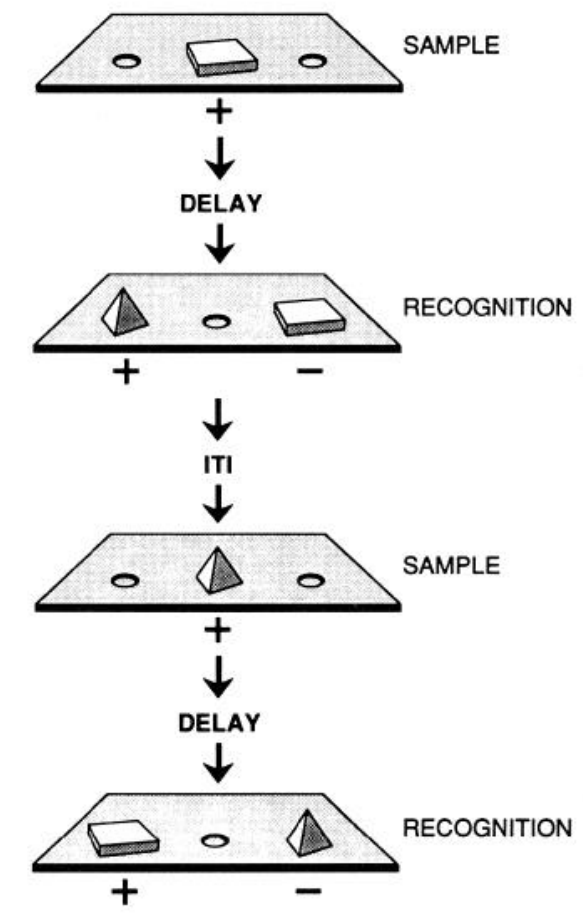

Figure 5. Schematic representation of the delayed nonmatching to sample task with trial repeated objects (DNMS-RO). The same pair of objects was used on every trial, and subjects were required to remember which object was presented most recently (i.e., before the delay interval) in order to perform correctly $(+$, baited, - , unbaited). Monkeys were initially trained to a learning criterion with a 10-sec delay. Memory for the sample object was subsequently tested across delays of 15-60 sec.

keys are only impaired at longer retention intervals is important because it suggests that age-related DR deficits are specifically related to the memory demands of the task. Subsequent work has demonstrated that these deficits persist over extensive periods of training, indicating that DR impairments are not related to test sophistication in aged subjects (Marriott and Abelson, 1980). Although other laboratories have reported that aged monkeys also fail to learn DR tasks conducted in the WGTA with delays as short as $5 \mathrm{sec}$ (Arnsten and Goldman-Rakic, 1985a; Walker et al., 1988), the age $\times$ delay interaction that characterizes the performance of aged animals in automated versions of this task was not observed in these earlier studies. The present report, however, provides a clear demonstration that aged monkeys exhibit reliable and robust delay-dependent deficits in the experimenter-paced, WGTA version of the DR task. These findings are therefore consistent with the view that delay-dependent DR impairments are a prominent feature of cognitive dysfunction in the aged monkey.

Two laboratories have reported that aged monkeys are significantly impaired in a trial unique objects version of DNMS similar to the one used here (Presty et al., 1987; Moss et al., 1988). Although the difference in age between the young and the oldest groups was even greater than in the present investigation, the degree of impairment in both earlier studies was modest. Moreover, neither investigation found aged subjects to be disproportionately impaired when the memory demands of the DNMS-TU task were manipulated by increasing the retention interval. The absence of an interaction between age and retention interval is important because delay-dependent deficits are a hallmark feature of memory impairments produced by a variety of experimental manipulations including medial temporal lobe ablation. It is important to consider the possibility, however, that the parametric features of the typical DNMS-TU procedure limit its sensitivity to age-dependent memory dysfunction (Rapp, 1988). In previous studies, aged monkeys were tested in the DNMS-TU task using only those retention intervals (i.e., $5 \mathrm{~min}$ or less) at which medial temporal lobe-damaged subjects exhibit severe deficits (Presty et al., 1987; Moss et al., 1988). That aged monkeys exhibit only mild impairments at these same delays should not be surprising since amnesia is not characteristic of normal aging. Previous studies were therefore unable to address the possibility that aged subjects would show more pronounced DNMS-TU deficits at retention intervals much longer than those used to investigate lesion effects in young subjects. In other words, earlier reports may have failed to note significant delay-dependent DNMS-TU deficits in aged monkeys because the memory demands of the testing procedures used were insufficient. In the present study, however, recognition memory was tested at much longer delays (up to $22 \mathrm{hr}$ ) where even the younger subject's performance deteriorated. Based on these findings, it seems clear that the lack of delay-dependent DNMS-TU impairments in aged monkeys is reliable across retention delays spanning virtually the entire forgetting curve for object recognition memory. These results are therefore consistent with earlier reports and provide additional evidence that recognition memory is, at most, only slightly impaired in the aged monkey.

These findings demonstrate that robust DR deficits in the aged monkey occur against a background of largely preserved functions assessed by the DNMS-TU task. The intact performance of aged subjects during postacquisition DNMS-TU test-
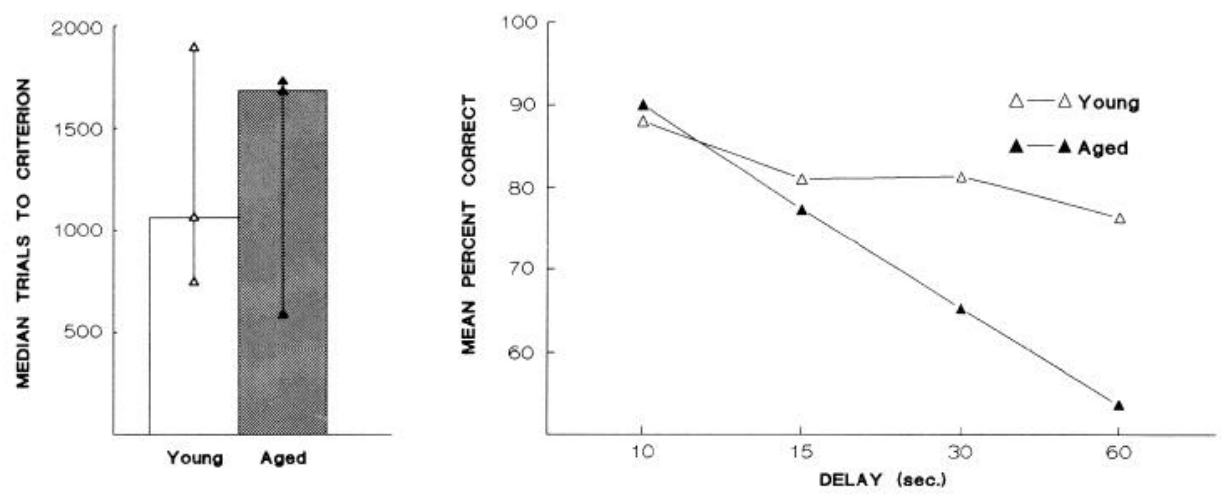

Figure 6. Performance of young $(n=$ $3)$ and aged $(n=3)$ monkeys on the delayed nonmatching to sample task with trial repeated objects (DNMS-RO). Left, Median number of trials to reach criterion for subjects who learned the DNMS-RO task. One young subject performed at only $83 \%$ correct after 1900 trials using a 10-sec delay. Symbols represent the learning scores for individual animals. Right, Average percent correct of the young and aged groups during DNMS-RO testing with delays of $10-60 \mathrm{sec}$. 
ing and during DR training with short delays suggests that global nonspecific deterioration is not a prominent feature of senescence in the monkey. Robust age-related changes in motivation or attention, for example, would be expected to result in performance deficits across a variety of testing procedures irrespective of the memory demands of the tasks. Similarly, sensory deficits in aged subjects, such as reduced visual acuity, would likely impair performance in any sufficiently difficult visually dependent testing procedure. Age-dependent change in nonspecific performance variables, therefore, cannot easily account for the observation that behavioral deficits in aged monkeys are both task specific and directly related to the memory demands of the testing procedures.

In contrast to the idea that age-related behavioral impairments result from nonspecific deterioration, DR deficits in aged monkeys have been interpreted as evidence for a decline in memory for recent stimulus events (for recent reviews, see Rupniak and Iversen, 1987; Dean and Bartus, 1988). The findings reported here, however, suggest that age-dependent memory dysfunction may be more selective than is implied by this interpretation. The object recognition capacities of aged monkeys, for example, indicate that not all forms of memory for recent stimulus events are affected adversely during aging. Reports that aged monkeys perform well in color, object, and pattern discrimination tasks provide additional evidence that not all mnemonic processes are equally affected during aging (Bartus et al., 1979; Arnsten and Goldman-Rakic, 1985a; Moss et al., 1988; Walker et al., 1988). These findings suggest that memory for specific types of information or specific aspects of mnemonic or cognitive processing may be differentially susceptible to agedependent deterioration.

This question was addressed in the present experiments by comparing the performance of aged monkeys across 2 parametric manipulations of the DNMS procedure. In contrast to their relatively intact performance on the trial unique objects version of DNMS, aged monkeys exhibited robust delay-dependent deficits when the same pair of objects was presented on every trial. Although both procedures share many identical task demands, DNMS-RO, unlike DNMS-TU, involves a recency discrimination; subjects must remember which object was presented more recently in order to perform accurately on the recognition phase of each trial. Since a general recognition memory deficit would lead to impairments on both tasks, the selective effects of aging on DNMS-RO may relate to the temporal memory requirements associated with this version of testing. In this context, it is interesting to note that the DR task entails a similar temporal memory requirement since, in this procedure, subjects are required to remember which food well was baited most recently. A selective age-dependent temporal memory deficit could therefore underlie the poor performance of older monkeys on both DR and DNMS-RO tasks. Alternatively, while DNMS$\mathrm{RO}$ deficits in aged monkeys may be related to the temporal memory requirements of this task, DR deficits in the same monkeys could reflect an age-dependent dysfunction in memory for spatial locations. The validity of this latter interpretation can be evaluated in future studies by determining if aged monkeys fail other spatial memory tasks that lack the temporal memory requirement of DR and DNMS-RO.

An alternative interpretation of the findings reported here is based on the proposal that aging in the monkey is accompanied by an increased sensitivity to interference (Bartus and Dean, 1979). On each trial in DR and DNMS-RO, subjects are re- quired to remember 1 of only 2 possible spatial locations (in DR) or objects (in DNMS-RO) so that there is a substantial opportunity for proactive interference between trials. In contrast, interference is relatively less pronounced in the DNMSTU task where novel stimuli are presented on every trial. According to this perspective, task-dependent deficits across these procedures might reflect this differential opportunity for interference rather than an agc-rclatcd impairment in memory for specific types of information. Several aspects of the results obtained, however, are difficult to reconcile with this interpretation. In both DR and DNMS-RO tasks, aged subjects were disproportionately impaired when the memory demands of the tasks were increased by lengthening the retention interval. Since interference is presumably operative in these tasks both on trials with short and longer retention intervals, an increased sensitivity to interference cannot easily account for the observation that aged monkeys were only impaired when tested with longer delays. The results of initial task acquisition also provide evidence inconsistent with an interference hypothesis since, of the 3 tasks used, aged subjects were reliably impaired during original training only in the low interference DMNS-TU procedure. Based on these considerations, it would appear that an increased sensitivity to interference cannot account for the pattern of agedependent cognitive deficits we have observed. Thesc findings, however, are not inconsistent with the view that, in other testing procedures, a hypersensitivity to interference may be an important component of the deficits aged monkeys exhibit (Bartus and Dean, 1979).

A neuropsychological perspective affords a valuable framework for efforts to identify the neural basis of age-related cognitive dysfunction. The behavioral assessment conducled here provides strong evidence that prominent aspects of memory dysfunction in the aged monkey are mediated by neural structures other than those located in the medial temporal lobe. In young monkeys, large medial temporal lobe lesions result in impairments on a variety of memory tasks, including DR and DNMS-TU (Zola-Morgan and Squire, 1985a; Zola-Morgan et al., 1989), and these findings are thought to reflect the critical importance of this neural system in the formation of memory for many types of information (Mishkin, 1982; Squire, 1987). As the present results demonstrate, however, DR deficits occur in aged monkeys against a background of relatively intact recognition memory. The modest impairment aged monkeys do show in DNMS-TU is not characterized by the sensitivity to retention delay that is the hallmark of medial temporal lobe damage (Mishkin, 1978; Zola-Morgan and Squire, 1985a). It is also important to cmphasizc that DR deficits in aged monkeys are more severe than can be accounted for by medial temporal lobe damage. Aged animals are dramatically impaired in this task at relatively short delays at which subjects with medial temporal lobe lesions perform normally (Orbach et al., 1960; Zola-Morgan and Squire, 1985a; Zola-Morgan et al., 1989). Although these findings leave open the possibility that medial temporal lobe dysfunction may contribute to age-related memory impairment, it is nonetheless clear that prominent features of cognitive dysfunction in the aged monkey must be mediated by other brain regions.

Based on comparisons with neuropsychological findings from young subjects, Bartus et al. have suggested that age-related cognitive deficits in the monkey are associated with prefrontal cortical dysfunction (Bartus, 1979; Bartus and Dean, 1979). Previous studies have noted that DR deficits in aged monkeys 
are qualitatively similar to the effects of prefrontal cortical damage in young subjects (Bartus, 1979; Arnsten and GoldmanRakic, 1985a, b). The effects of aging and dorsolateral prefrontal damage are also similar in that DNMS-TU performance remains largely intact in both cases (Bachevalier and Mishkin, 1986). A novel component of the approach adopted here is that hypotheses concerning the neural systems that are responsible for specific features of senescent memory impairment can be used to formulate testable predictions concerning the behavioral tasks that should be sensitive to that damage. Current theories, for example, emphasize that the prefrontal cortex is critically involved in the temporal organization of memory (Fuster, 1985; Milner et al., 1985; Schacter, 1987). This interpretation leads to the prediction that prefrontal cortical dysfunction in aged monkeys should be reflected as behavioral impairments that are related to the temporal memory demands of a given testing procedure. In confirmation of this prediction, aged monkeys were selectively impaired on DNMS procedures when they were required to remember the temporal order in which objects were presented. The relationship of these age-associated behavioral deficits to prefrontal cortical dysfunction is further supported by the observation that, in testing procedures similar to the DNMS tasks used here, young monkeys with lateral prefrontal cortical damage are selectively impaired in a repeated objects recognition memory paradigm (Mishkin and Manning, 1978; Bachevalier and Mishkin, 1986).

The findings of the present report support the use of the aged nonhuman primate as a model of normal human aging. A number of groups have reported that DR tasks and conceptually similar procedures are particularly sensitive to aging in nondemented humans (see Dean and Bartus, 1988, for a recent review). Recent evidence suggesting that the temporal organization of memory is compromised as a consequence of aging in humans (Janowsky et al., 1989) also is consistent with the results of the present studies. In contrast, the degree to which recognition memory is affected during normal human aging remains a matter of some controversy (Schonfield and Robertson, 1966; Erber, 1974; Ferris et al., 1980; Rabinowitz, 1984). As in the aged monkey, when significant age-dependent recognition memory impairments have been observed in humans, the deficits are not exacerbated when retention is measured over increasing memory delays (Ferris et al., 1980). Since many of the neuropathological changes associated with human aging (i.e., regionspecific cell loss and neuritic plaque formation) also are seen in the aged monkey brain (Brizzee et al., 1976; Ball et al., 1983; Struble et al., 1985), it is possible that parallel behavioral deficits in aged monkeys and humans may share a common neural basis. The aged monkey may, therefore, provide a unique model system for investigating the relationship between neural alterations and cognitive dysfunction in aging.

\section{References}

Adamowicz, J. K. (1976) Visual short-term memory and aging. J Gerontol. 31: 39-46.

Aggleton, J. P., and M. Mishkin (1983a) Visual recognition impairment following medial thalamic lesions in monkeys. Neuropsychologia 21: 189-197.

Aggleton, J. P., and M. Mishkin (1983b) Memory impairment following restricted medial thalamic lesions in monkeys. Exp. Brain Res. 52: 199-209.

Arnsten, A. F. T., and P. S. Goldman-Rakic (1985a) Catecholamines and cognitive decline in aged nonhuman primates. Ann. NY Acad. Sci. 444: 218-241.
Arnsten, A. F. T., and P. S. Goldman-Rakic (1985b) Alpha ${ }_{2}$-adrenergic mechanisms in prefrontal cortex associated with cognitive decline in aged nonhuman primates. Science 230: 1273-1276.

Bachevalier, J., and M. Mishkin (1986) Visual recognition impairment follows ventromedial but not dorsolateral prefrontal lesions in monkeys. Behav. Brain Res. 20: 249-261.

Ball, M. J., J. MacGregor, I. M. Fyfe, S. I. Rapoport, and E. D. London (1983) Paucity of morphological changes in the brains of ageing beagle dogs: Further evidence that Alzheimer lesions are unique for primate nervous system. Neurobiol. Aging 4: 127-131.

Barnes, C. A. (1979) Memory deficits associated with senescence: A neurophysiological and behavioral study in the rat. J. Comp. Physiol. Psychol. 93: 74-104.

Barnes, C. A., L. Nadel, and W. K. Honig (1980) Spatial memory deficit in senescent rats. Can. J. Psychol. 34: 29-39.

Bartus, R. T. (1979) Effects of aging on visual memory, sensory processing, and discrimination learning in a nonhuman primate. In Sensory Systems and Communications in the Elderly, Aging, Vol, 10, J. M. Ordy and K. Brizzee, eds., pp. 85-114, Plenum, New York.

Bartus, R. T., and R. L. Dean (1979) Recent memory in aged nonhuman primates: Hypersensitivity to visual interference during retention. Exp. Aging Res. 5: 385-400.

Bartus, R. T., D. Fleming, and H. R. Johnson (1978) Aging in the rhesus monkey: Debilitating effects on short-term memory. J. Gerontol. 33: 858-871.

Bartus, R. T., R. L. Dean, and D. L. Fleming (1979) Aging in the rhesus monkey: Effects on visual discrimination learning and reversal learning. J. Gerontol. 34: 209-219.

Beatty, W. W., D. P. Salmon, N. Butters, W. C. Heindel, and E. L. Granholm (1988) Retrograde amnesia in patients with Alzheimer's disease or Huntington's disease. Neurobiol. Aging 9: 181-186.

Brizzee, K. R., J. M. Ordy, J. Hansche, and B. Kaack (1976) Quantitative assessment of changes in neuron and glia cell packing density and lipofuscin accumulation with age in the cerebral cortex of a nonhuman primate (Macaca mulatta). Neurobiol. Aging 3: 229-244.

Davis, R. T. (1985) The effects of aging on the behavior of rhesus monkeys. In Behavior and Pathology of Aging in Rhesus Monkeys, R. T. Davis and C. W. Leathers, eds., pp. 57-82, Alan R. Liss, Inc., New York.

Dean, R. L., and R. T. Bartus (1988) Bchavioral modcls of aging in nonhuman primates. In Handbook of Psychopharmacology, Vol. 20, L. L. Iversen, S. D. Iversen, and S. H. Snyder, eds., pp. 325-392, Plenum Press, New York.

Erber, J. T. (1974) Age differences in recognition memory. J. Gerontol. 29: $177-181$.

Eslinger, P. J., and A. R. Damasio (1986) Preserved motor learning in Alzheimer's disease: Implications for anatomy and behavior. J. Neurosci. 6: 3006-3009.

Ferris, S. H., T. Crook, E. Clark, M. McCarthy, and D. Rae (1980) Facial recognition memory deficits in normal aging and senile dementia. J. Gerontol. 35: 707-714.

Flicker, C., R. T. Bartus, T. H. Crook, and S. H. Ferris (1984) Effects of aging and dementia upon recent visuospatial memory. Neurobiol. Aging 5: 275-283.

Fuster, J. M. (1985) The prefrontal cortex, mediator of cross-temporal contingencies. Human Neurobiol. 4: 169-179.

Gaffan, D., and S. Harrison (1987) Amygdalectomy and disconnection in visual learning for auditory secondary reinforcement by monkeys. J. Neurosci. 7: 2285-2292.

Gage, F. H., S. S. Dunnett, and A. Bjorklund (1984) Spatial learning and motor deficits in aged rats. Neurobiol. Aging 5: 43-48.

Goldman-Rakic, P. S. (1987) Circuitry of the prefrontal cortex and regulation of behavior by representational memory. In Handbook of Physiology, Vol. 5, V. B. Mountcastle, F. Plum, and S. R. Geiger, eds., pp. 373-417, Williams \& Wilkins, Baltimore, MD.

Goo, G. P. (1986) Primate colony management of harem breeding groups of rhesus monkeys (Macaca mulatta). In Current Perspectives in Primate Biology, D. M. Traub and F. A. King, eds., pp. 71-78, Van Nostrand Reinhold, New York.

Harlow, H. F., and J. A. Bromer (1938) A test apparatus for monkeys. Psychol. Rec. 2: 434-436.

Heindel, W. C., D. P. Salmon, C. W. Shults, P. A. Walicke, and N. Butters (1989) Neuropsychological evidence for multiple implicit memory systems: A comparison of Alzheimer's, Huntington's and Parkinson's disease patients. J. Neurosci. 9: 582-587. 
Janowsky, J. S., A. P. Shimamura, and L. R. Squire (1989) Source memory impairments in patients with frontal lobe lesions. Neuropsychologia (in press)

Light, L. L., A. Singh, and J. L. Capps (1986) Dissociation of memory and awareness in young and older adults. J. Clin. Exp. Neuropsychol. 8: $62-74$.

Marriott, J. G., and J. S. Abelson (1980) Age differences in short-term memory in test-sophisticated rhesus monkeys. Age 3: 7-9.

Medin, D. L. (1969) Form perception and pattern reproduction by monkeys. J. Comp. Physiol. Psychol. 68: 412-419.

Milner, B., and M. Petrides (1984) Behavioural effects of frontal-lobe lesions in man. Trends Neurosci. 7: 403-407.

Milner, B., M. Petrides, and M. L. Smith (1985) Frontal lobes and the temporal organization of memory. Human Neurobiol. 4: 137142.

Mishkin, M. (1978) Memory in monkeys severely impaired by combined but not separate removal of amygdala and hippocampus. Nature 273: 297-298.

Mishkin, M. (1982) A memory system in the monkey. Proc. R. Soc. Lond. [Biol.] 298: 85-95.

Mishkin, M., and F. J. Manning (1978) Non-spatial memory after selective prefrontal lesions in monkeys. Brain Res. 143: 313-323.

Moss, M. B., D. L. Rosene, and A. Peters (1988) Effects of aging on visual recognition memory in the rhesus monkey. Neurobiol. Aging 9: 495-502.

Murray, E. A., and M. Mishkin (1985) Amygdalectomy impairs crossmodal association in monkeys. Science 228: 604-606.

Orbach, J., B. Milner, and T. Rasmussen (1960) Learning and retention in monkeys after amygdala-hippocampal resection. Arch. Neurol. 3: 230-251.

Park, D. C., J. T. Puglisi, and M. Sovacool (1983) Memory for pictures, words, and spatial location in older adults: Evidence for pictorial superiority. J. Gerontol. 38: 582-588.

Pelleymounter, M. A., M. Y. Smith, and M. Gallagher (1987) Spatial learning impairments in aged rats trained with a salient configuration of stimuli. Psychobiology 15: 248-254.

Perlmutter, M., R. Metzger, T. Nezworski, and K. Miller (1981) Spatial and temporal memory in 20 and 60 year olds. J. Gerontol. 36: 5965.

Presty, S. K., J. Bachevalier, L. C. Walker, R. G. Struble, D. L. Price, M. Mishkin, and L. C. Cork (1987) Age differences in recognition memory of the rhesus monkey (Macaca mulatta). Neurobiol. Aging 8: $435-440$.
Rabinowitz, J. C. (1984) Aging and recognition failure. J. Gerontol. 39: 65-71.

Rapp, P. R. (1988) Toward a nonhuman primate model of age-dependent cognitive dysfunction. Neurobiol. Aging 9: 503-505.

Rapp, P. R., R. Rosenberg, and M. Gallagher (1987) An evaluation of spatial information processing in the aged rat. Behav. Neurosci. 101: 3-12

Rupniak, N. M. J., and S. D. Iversen (1987) Primate models of senile dementia. In Cognitive Neurochemistry, S. M. Stahl, S. D. Iversen, and E. C. Goodman, eds., pp. 57-72, Oxford U. P., Oxford.

Sagar, H. J., N. J. Cohen, E. V. Sullivan, S. Corkin, and J. H. Growdon (1988) Remote memory function in Alzheimer's disease and Parkinson's disease. Brain 111: 185-206.

Schacter, D. L. (1987) Memory, amnesia, and frontal lobe dysfunction. Psychobiology 15: 21-36.

Schonfield, D., and B. Robertson (1966) Memory storage and aging. Can. J. Psychol. 20: 228-236.

Scoville, W. B., and B. Milner (1957) Loss of recent memory after bilateral hippocampal lesions. J. Neurol. Psychiatr. 20: 11-21.

Squire, L. R. (1987) Memory and Brain, Oxford U. P., New York.

Squire, L. R., and R. Y. Moore (1979) Dorsal thalamic lesions in a noted case of human memory dysfunction. Ann. Neurol. 6: 503-506.

Struble, R. G., D. L. Price, L. C. Cork, and D. L. Price, Jr. (1985) Senile plaques in cortex of aged normal monkeys. Brain Res. 361: 267-275.

Tigges, J., T. P. Gordon, H. M. McClure, E. C. Hall, and A. Peters (1988) Survival rate and life span of rhesus monkeys at the Yerkes Regional Primate Center. Am. J. Primatol. 15: 263-273.

Victor, M., R. D. Adams, and G. H. Collins (1971) The WernickeKorsakoff Syndrome, Davis, Philadelphia

Walker, L. C., C. A. Kitt, R. G. Struble, M. V. Wagster, D. L. Price, and L. C. Cork (1988) The neural basis of memory decline in aged monkeys. Neurobiol. Aging 9: 657-666.

Zola-Morgan, S., and L. R. Squire (1985a) Medial temporal lesions in monkeys impair memory on a variety of tasks sensitive to human amnesia. Behav. Neurosci. 99: 22-34.

Zola-Morgan, S., and L. R. Squire (1985b) Amnesia in monkeys after lesions of the mediodorsal nucleus of the thalamus. Ann. Neurol. 17: 558-564.

Zola-Morgan, S., L. R. Squire, and D. G. Amaral (1989) Lesions of the hippocampal formation but not lesions of the fornix or the mammillary nuclei produce long-lasting memory impairment in monkeys. J. Neurosci. 9: 898-913. 